\title{
Road Network Extraction from Remote Sensing using Region-based Mathematical Morphology
}

\author{
Luc Courtrai and Sébastien Lefèvre \\ Univ. Bretagne-Sud, UMR 6074 IRISA \\ F-56000, Vannes, France \\ Email: \{luc.courtrai, sebastien.lefevre\}@irisa.fr
}

\begin{abstract}
In this paper, we introduce an efficient and automatic method for road extraction from satellite or aerial images. It builds upon an existing work based on (incomplete) path opening/closing, morphological filters able to deal with curvilinear structures. We propose here to apply such techniques not on pixels directly but rather on regions representing road segments, to improve both efficiency and robustness. To do so, we map road segments by rectangular areas, which are identified in a first step. We then adapt the morphological filters to process regions instead of pixels. The resulting path filter allows for connection of road segments, in order to identify roads of minimal length. Robustness to occlusion is ensured through the adaptation of the incomplete strategy to our context, while better discrimination between road segments and other objects relies on background knowledge through an hit-or-miss transform. Preliminary results obtained on several satellite images are promising.
\end{abstract}

\section{Introduction}

Informations related to road networks are of high importance for urban planning or environmental monitoring. While road networks are often modeled into GIS, their extraction from remote sensing data eases GIS updating (on a regular basis or after a disaster) and is required when no GIS is available. However, automatic extraction of road networks from aerial or satellite images is still very challenging (see for instance results from the ISPRS Test Project on Urban Classification and 3D Building Reconstruction [1]). Among the possible techniques which have shown their relevance in this context, mathematical morphology leads to promising results. Indeed, path opening and closing are morphological filters which aim to extract (or highlight) curvilinear structures [2]. Very unsurprisingly, their recent use in remote sensing for extracting road networks was successful [3]. However, this technique (as most of the automatic techniques) is relying on pixelwise analysis and filtering, thus presenting two drawbacks. On the one side, the volume of information to be processed (pixels) prevents from efficient (fast) extraction and does not allow to process large remotely-sensed images. On the other side, it is not adapted to images with a very high (spatial) resolution where roads are described by a large set of pixels (e.g., a road of $7 \mathrm{~m}$ width is mapped by image segments of 10 to 100 pixels wide for spatial resolutions of $0.7 \mathrm{~m}$ to $0.07 \mathrm{~m}$ per pixel, corresponding respectively to VHR satellite and aerial images).

We build here upon [3] and introduce a novel technique for road network extraction from remote sensing. We also rely on mathematical morphology but, conversely to [3], we consider here the region level instead of the (standard) pixel one. Thus we propose a 2-step approach which first extracts rectangular regions corresponding to possible road segments, before connecting these regions through regionbased morphological operators. Our contribution also consists in adapting the (incomplete) path opening paradigm from [2] to a region-based representation of the image, and also in taking into account background knowledge through an HMT (hit-or-miss transform) procedure similarly to [4]. Preliminary results show the relevance of the method for automatic and efficient extraction of the road network, but also its improvement over the state-of-the-art (in particular [3]) when dealing with roads of more than 1-pixel width.

Our paper is organized as follows: Section 2 provides a description of the method through its different steps. Results obtained on several images are provided in Section 3, as well as a comparison with the state-of-the-art through qualitative and quantitative evaluation. Section 4 concludes this paper and discusses future research directions.

\section{Method}

The proposed method is made of two main steps: extraction and connection of road segments. Robustness to false positive and negative is ensured through two additional steps: filtering with background knowledge and reconstruction of incomplete segments. Figure 1 shows the overall flowchart.

\subsection{Extraction of road segments}

Existing methods for road extraction from remote sensing data usually operate at the pixel scale. However, such a scale requires a high volume of data to be processed and thus prevents scalability. Furthermore, it is not well adapted with very high (spatial) resolution, where road width varies from 10 to 100 pixels. We address these two issues by introducing a preprocessing step, the goal of which is to change the 
analysis scale from pixels to regions. These regions are identified within the image as possible road segments. We rely here on the assumption that a road segment corresponds to a rectangular area of relative homogeneous content.

The extraction of road segments is then achieved through a probing step. It requires the definition of a rectangular template of predefined size (height and width), which is used as a sliding window when scanning the image. For each pixel, the template leads to the identification of its neighbors. The pixel is then compared with each neighbor, and the ratio of similar pixels within the template is computed. If this ratio is higher than a predefined threshold, we consider that a possible road segment is present.

\subsection{Connection of road segments}

Once road segments have been identified, they are used as the elementary units of the image for further processing. We rely here on morphological filters, and more precisely on path opening and closing [2] able to extract curvilinear structures and that led recently to promising results for road extraction [3]. Indeed, it is possible to distinguish between a road (i.e., a chain of segments with significant length) and a building (an unordered set of segments) while these objects can share the same spectral properties.

We thus adapt the algorithms from [2] to process segments instead of pixels. The original algorithm builds paths by linking neighboring pixels depending on their graylevel and following a given direction. We define here the neighborhood of a given segment $S$ as the set of all segments overlapping $S$ and having a grayscale lower or equal to the one of $S$. To ensure robustness, we relax this definition by also considering segments relatively close to $S$ (distance between segments lower than a threshold), and with a similar orientation (orientation difference lower than a threshold).

The filtering used in this paper is a closing: it will help to identify long dark paths, while all other structures will be brightened. The overall algorithm is applied iteratively on graylevels, from the lowest (black) to the highest (white) levels. For each graylevel $l$, paths are built from segments with graylevel lower or equal to $l$. Each segment is set to graylevel $l$ if it belongs to a path with length higher than a threshold. Thus it will be kept unchanged if it is possible to build a path of significant length from its graylevel. Otherwise, it will necessarily belong to a path of higher graylevel, and thus will have its graylevel set accordingly.

So the road segments contained in the filtered image have their graylevels brightened depending on the size of the longest path they belong to. Thresholding this filtered image allows to produce the final road network map.

\subsection{Filtering with background knowledge}

Accuracy of the road network map directly relies on the quality of road segments extracted in the first step. Among these segments, a significant subset corresponds to large and homogeneous areas (e.g., parking lots, courts, fields, etc.) and leads to false positive. We propose to take into account
Figure 1. Flowchart of the proposed method.

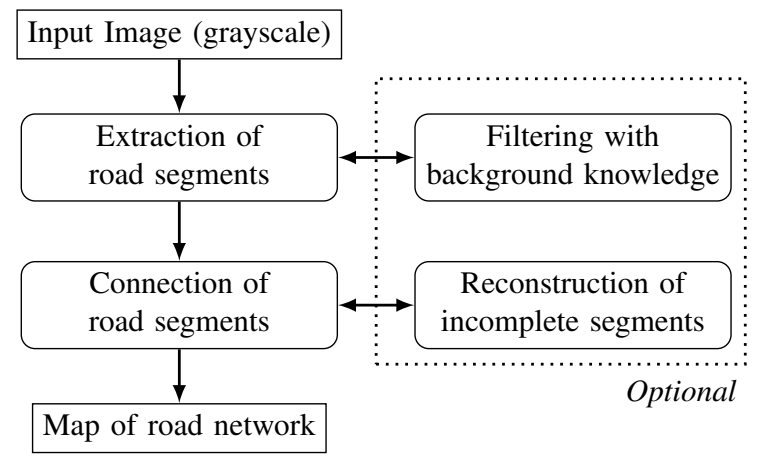

background knowledge for a more accurate identification of road segments. We thus follow the principle of the Hit-orMiss Transform (HMT) that led to the successful design of template matching solutions for a wide range of problems, including in remote sensing [4]. Assuming that roads can be distinguished from other areas by their width, we modify the first step to take into account both a foreground rectangular template and a composite background template. The latter is made of two parallel rectangular areas located on each side of the foreground template. Distance between these templates and the foreground one is set depending on the expected road width and its possible variation (e.g., varying number of lanes). The pixels not contained in the foreground nor the background templates (i.e., in-between area) corresponds to an uncertainty zone, and are not involved in the template matching process. The probing now operates on two criteria: all pixels covered by the foreground (resp. background) template shall have a similar (resp. different) value to the central pixel, i.e., foreground (resp. background) color.

\subsection{Reconstruction of incomplete segments}

While the previous step aims to remove false positive, it is also necessary to remove false negative. Indeed, some roads might not be completely detected due to a partial occlusion (e.g., by trees, shadow, cars, pedestrian crossing, etc.) of road segments, leading to their heterogeneous content and a subsequent missed detection. This in turn results in roads of insufficient length, discarded by the path closing process.

Similarly to [2], we also propose incomplete path opening/closing to deal with missing elements (but here, elements are segments instead of pixels). A scanning process is started for each road extremity. It tries to fill with a small set of successive segments the possible gap between the current road and another one. To ensure coherence between the added segments and the original ones, and to avoid the inclusion of outliers (e.g., a building located between two roads), the connecting segments shall share some common properties. Here we rely on the same criteria than in the path closing step, but using some lowered constraints (thresholds). This reconstruction process is included in the general path closing step, thus enabling to connect road segments even in presence of missing segments. 


\section{Experiments}

\subsection{Dataset}

To assess our contribution, we have performed a series of experiments on satellite Quickbird images: a grayscale image of $420 \times 300$ pixels used in [3], and a multispectral image of $2832 \times 2772$ pixels from Strasbourg, France. The latter has been converted to grayscale and is associated with some validation data provided by French Geographic Institute IGN.

Evaluation is performed using standard completeness (recall) and correctness (precision), as well as a quality score. The latter is defined using $T P, F P, F N$ (respectively denoting true positive, false positive, and false negative) by the ratio $T P /(T P+F P+F N)$.

\subsection{Parameter settings}

The method proposed in this paper relies on a number of parameters that have been set empirically from image properties (e.g., spatial resolution, contrast). In the experiments reported here (Strasbourg image), we have used the following settings. Road segments have width of 5 pixels, and height of 45 pixels. Segments are detected in all orientations with an angular step of $5^{\circ}$. Maximal orientation change and maximal distance between segments are respectively set to $30^{\circ}$ and 5 pixels. Pixels are considered similar if their graylevel are equal $\pm 5 \%$. The rate of similar pixels within a segment is set to $100 \%$ (all pixels shall be similar). Background knowledge requires the definition of an uncertainty zone in the HMT process. It has been set here equals to road width (5 pixels). Reconstruction fills gaps of length once or twice the size of segments (i.e., 45 or 90 pixels). A path is considered as a road if it contains at least 5 successive segments (ca. 250 pixels). Finally, pixels from the filtered image are kept in the road network map if their graylevel is lower to 128 . Since we consider asphalt roads, the final map contains the dark half of the filtered image, while the other objects are made of pixels brightened by the region-based morphological filtering).

While parameters have been set here empirically, the indepth analysis of their impact on the end-results, as well as their automatization, are left for future work. Indeed, the parameters greatly depend on the image properties but also on the kind of road network considered, since the latter may vary considerably from one country to the other, and even within a given country (urban versus rural areas).

\subsection{Results}

We compare our method with some representative techniques from the state of the art: the road extraction algorithm available in OTB (CNES ORFEO Tool Box, http: //www.orfeo-toolbox.org/otb) and the method from Valero et al. [3]. The latter has been here initialized manually with the parameters leading to the best results.

We first consider the easiest dataset already suggested in [3]. We can observe in Figure 2 that our method and [3] provide similar results, better than with OTB which does not

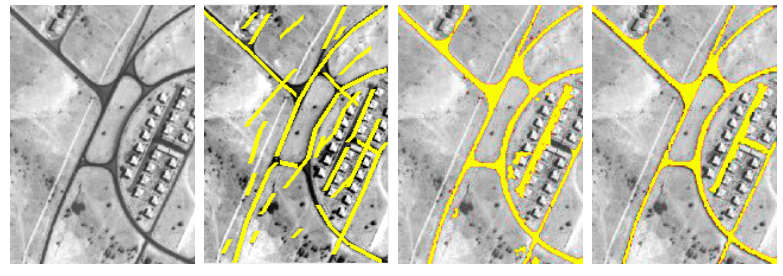

Figure 2. Road network extraction with an easy dataset: (from left to right) original image and results from OTB, Valero et al., and our method.

Table 1. Quantitative evaluation of road network extractors with an easy dataset.

\begin{tabular}{lccc}
\hline Method & Completeness & Correctness & Quality \\
\hline OTB & 0.63 & 0.70 & 0.50 \\
Valero et al. & $\mathbf{0 . 9 5}$ & 0.78 & 0.76 \\
Proposed & 0.93 & $\mathbf{0 . 8 5}$ & $\mathbf{0 . 8 1}$ \\
\hline
\end{tabular}

Table 2. Quantitative evaluation of road network extractors with a challenging dataset.

\begin{tabular}{cccc}
\hline Method & Completeness & Correctness & Quality \\
\hline Baseline & 0.68 & 0.80 & 0.58 \\
Baseline & 0.70 & 0.82 & 0.61 \\
+ Filtering & 0.69 & 0.81 & 0.60 \\
Reconstruction & $\mathbf{0 . 7 1}$ & $\mathbf{0 . 8 3}$ & $\mathbf{0 . 6 2}$ \\
$\begin{array}{c}\text { Reconstruction } \\
\text { + Filtering }\end{array}$ & & & \\
\hline
\end{tabular}

rely on paths. A quantitative analysis is provided in Table 1, confirming the conclusions driven from visual analysis.

A second and more challenging experiment has been performed with the Strasbourg image. We can see in Figure 3 (a subset of the original image for better visualization) that roads have a very similar color to the background. While such a difficult scenario cannot be tackled by [3] (nor by the OTB method which is unable to analyze such a complex image), our method (with all steps from Figure 1) is able to extract the most important elements of the road network. We clearly observe the relevance of mapping road segments by rectangular areas, allowing to distinguish between road segments and neighboring pixels of similar color.

Once roads have been extracted, the availability of some reference data provided by IGN allows us to perform a quantitative evaluation. Only a part of the Strasbourg image is available with some ground truth (Figure $3(a), 1000 \times 1000$ pixels) and leads to quantitative evaluation. Table 2 gives the results obtained with our baseline method, as well as alternative steps to improve robustness to false positive/negative. We can quantitatively assess the relevance of these options.

Beyond a major improvement in detection accuracy, the proposed region-based morphological scheme also allows a significant decrease of computation time. Measures obtained with the full Strasbourg image are reported in Table 3. Let us observe that our method requires 22 seconds to extract road segments, which lowers the efficiency gain if no reconstruction is performed. However, processing regions 

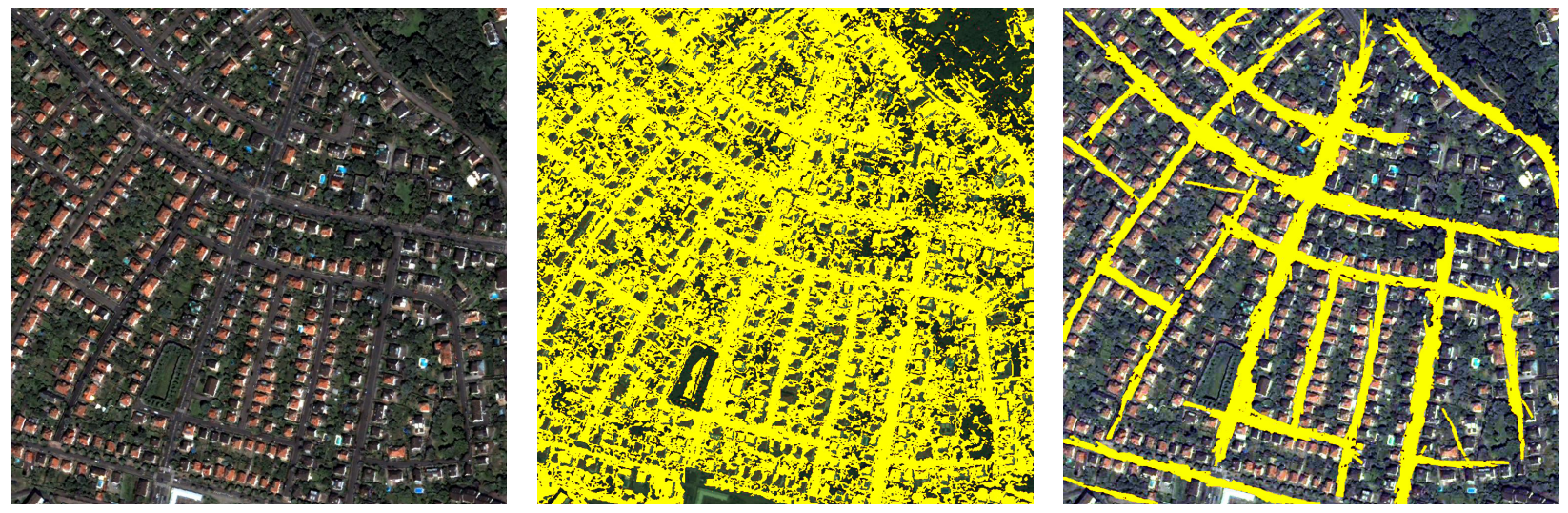

Figure 3. Road network extraction with a challenging dataset: (from left to right) RGB composition of the original image (C)Digitalglobe 2008, and results from Valero et al. and our method.

Table 3. Comparison of processing times (in seconds).

\begin{tabular}{lcr}
\hline Method & Reconstruction & CPU Time \\
\hline Valero et al. & no & 98 \\
Valero et al. & 1 pixel & 310 \\
Valero et al. & 2 pixels & 720 \\
Proposed & no & 84 \\
Proposed & 1 segment & 117 \\
Proposed & 2 segments & 119 \\
\hline
\end{tabular}

instead of pixels leads to a major improvement in efficiency as soon as a reconstruction step is involved. Moreover, the complexity increases only slightly when considering gaps of higher length ( 1 or 2 segments, i.e., ca. 50 or 100 pixels). Let us note that these results have been obtained without any optimization, and thus shall be considered carefully.

\section{Conclusion}

Automatic road extraction from aerial or satellite imagery is a fundamental step to maintain an up-to-date map of road networks. While this problem has led to many studies in the past, it is still very challenging, especially when considering remote sensing data with very high spatial resolution. In this paper, we address this issue and propose to rely on regionbased mathematical morphology. The input image is mapped into road segments which become the elementary image units, following a template matching operation based on a Hit-or-Miss Transform. These segments are subsequently processed with an (incomplete) path closing algorithm.

The preliminary results obtained on several remotelysensed images are appealing. Indeed, the proposed method outperforms preliminary work based on path closing [3] both in terms of accuracy and efficiency. However, the method still needs to be evaluated with a larger dataset, e.g., the very challenging one provided by the ISPRS Test Project on Urban Classification and 3D Building Reconstruction [1].

Another future research direction is related to the use of the color/spectral information. Indeed, such information is currently not taken into account (the input image being con- verted to grayscale), while color/multispectral morphology is now a mature topic [5]. Beyond color, texture information can also be taken into account. Using color or texture would allow to consider roads composed of various material (e.g., concrete or rock) and not only of asphalt as addressed here.

\section{Acknowledgments}

The authors would like to thank A. Puissant from LIVE UMR CNRS 7362 (University of Strasbourg) for providing the geometrically corrected Quickbird image of Strasbourg.

The authors also acknowledge the support of the French Agence Nationale de la Recherche (ANR) under reference ANR-13-JS02-0005-01 (Asterix project).

\section{References}

[1] F. Rottensteiner, G. Sohn, J. Jung, M. Gerke, C. Baillard, S. Benitez, and U. Breitkopf, "The ISPRS benchmark in urban object classification and 3d building reconstruction," ISPRS Annals of Photogrammetry, Remote Sensing and Spatial Information Sciences, vol. I-3, pp. 293-298, 2012.

[2] H. Talbot and B. Appleton, "Efficient complete and incomplete path openings and closings," Image and Vision Computing, vol. 25, no. 4, pp. 416-425, 2007.

[3] S. Valero, J. Chanussot, J. Benediktsson, H. Talbot, and B. Waske, "Advanced directional mathematical morphology for the detection of the road network in very high resolution remote sensing images," Pattern Recognition Letters, vol. 31, no. 10, pp. 1120-1127, 2010.

[4] S. Lefèvre, E. Aptoula, B. Perret, and J. Weber, "Morphological template matching in color images," in Advances in Low-Level Color Image Processing, ser. Lecture Notes in Computational Vision and Biomechanics, E. Celbi and B. Smolka, Eds. Springer, 2014, vol. 11, pp. 241-277.

[5] E. Aptoula and S. Lefèvre, "A comparative study on multivariate mathematical morphology," Pattern Recognition, vol. 40, no. 11, pp. 2914-2929, 2007. 